but thoughtful and often provocative. Many questions are posed for the reader to ponder, where other authors might peddle their own pet speculations.

The book as a whole contains much that one might quibble with (I would particularly quarrel with Slack's definition of a stem cell); it does not offer revolutionary new insights; it is not exhaustive - the account of chemical reaction systems, for example, makes no mention of the possibility of chaotic behaviour. But it is entertainingly written; it gives a clear and refreshing perspective on a major problem; and, above all, it stimulates thought.

Julian Lewis is a Lecturer in the Department of Anatomy at King's College, London.

\section{An alternative to life}

\section{Paul Calvert}

An Introduction to Speciality Polymers.

Edited by Norio Ise and Iwao

Tabushi.

Cambridge University Press: 1983.

Pp.200. £22.50, \$42.50.

"SPECIALITY polymers", is a phrase which, like "pure research" changes its meaning with the context. In this case it means "polymers for non-mechanical applications". The field is enormous and this book is not a general survey but concentrates on synthetic polymer equivalents of active biological polymers including enzyme-like catalysts, photosynthetic systems, muscle-like polymers, polymers for selective transport, membrane formers and nucleic acid analogues. If you have been set the problem of creating an alternative form of life not based on proteins or nucleic acids, this is the book you need to begin.

It was originally published in Japanese in 1980 , but has been updated and the membrane chapter added for the English edition. After a brief introduction there is a chapter by Makoto Okawara on reactive polymers. This essentially describes the tool-kit, surveying methods for producing polymer chains with a variety of attached reactive groups. There is nothing particularly revolutionary or all-embracing here but it is a useful review of what can be done.

Norio Ise reviews with great clarity polymers as catalysts, discussing the different types of interaction between the catalyst and the reactants. A second part to the same chapter by Iwao Tabushi covers attempts to produce polymers with an enzyme-like function. He flounders a bit in discussing what enzymic catalysis actually is when contrasted with other homogeneous catalysis. There are a variety of examples of low molecular weight catalysts which also work when attached to polymers but no sign as yet of any specifically "large molecule" effect.

Energy-converting polymers are dealt with by Shigeo Tazuke, who starts with muscle-like systems which convert chemical, thermal or light energy into mechanical energy by undergoing a conformational change. Interestingly the power densities and forces developed seem to be similar to those of muscle though the only machines produced are in the category of intriguing toys. The implication would seem to be that biological design cannot compete in the energy-intensive artificial chemical and photoelectric systems, touching briefly on many topics, but is not a coherent discussion. Data from an American paper on energy storage have crept through the double translation in the units of yen $\mathrm{kg}^{-1}$. We need SI currency units.

Chapter Five by Manabu Senō gives a useful general survey of polymer membranes. He then goes on to talk about facilitated transport and active transport,

\section{News of the growth business}

\section{J. Taylor-Papadimitriou}

Growth and Maturation Factors, Vol.1. Edited by Gordon Guroff.

Wiley: 1983. Pp.365. £51.75, \$72.50.

Two of the major hazards facing authors writing a review on an expanding subject are, firstly, the possibility of new developments arising during the period from manuscript submission to publication and, secondly, the temptation to overemphasize work from the author's own laboratory. Although these hazards have not been completely avoided by the authors in Growth and Maturation Factors Vol.1, the book does present a fairly comprehensive and balanced view of the properties and functions of some classes of growth factors. The most obvious omission is the recent data relating platelet-derived growth factor (PDGF) to the sis gene from simian sarcoma virus which has been well publicized. On the other hand, this development justifies the idea of producing a set of volumes to "present the unfolding information".

The material in the first volume tends to be organized around individual, or classes of growth factors. Where the growth factors themselves are well characterized as with epidermal growth factor (EGF), tumour promoters, nerve growth factor and PDGF, more space is devoted to recent data on the mechanism of their action. In these chapters, review of the literature and presentation of recent data is well balanced ๑) 1983 Nature Publishing Group world. The rest of the chapter is on photo- largely in biological systems, where the permeant is carried through the membrane by another molecule. A chapter on synthetic bilayer membranes follows, but does not really have much to do with polymers. The final chapter discusses informationtransmitting polymers but does not get beyond a brief survey of nucleic acids and a few polymers with nucleosides attached to synthetic macromolecules. It really shows only that we have not got far.

This is apparently intended as a textbook but it is not really suitable for a polymer course; the approach is very biological and very variable. As a research book it is unsatisfactory, and misses out too much. The references are mostly general and many workers are mentioned with no reference at all. Part of this may be due to a desire to avoid referring to publications in Japanese. Despite these drawbacks there is a great deal here to stimulate thought. The patchiness of the treatment leaves one with a feeling of how much more could be done.

Paul Calvert is in the School of Chemistry and Molecular Sciences, University of Sussex.

and interesting. In each case, the area of active research is given emphasis so that the structure, processing and in vivo action of nerve growth factor complements the studies presented on membrane interactions of tumour promoters and internalization of EGF in culture systems. Clearly, the in vitro model systems using defined media described in the book are useful and productive for studying mechanisms of action of specific growth factors, but most studies have been done with fibroblasts and the growth factors acting on them (including the so-called transforming factors and PDGF).

Where the growth factors are members of a class, the emphasis is on data characterizing the factors and their relationship to each other. Growth factors are generally named after the function they promote but this can be as vague as multiplication stimulating activity (MSA) or colony stimulating factor (CSF), which may describe the activity of many factors. Further clarification is essential to unravel the complexity of growth factor interactions in vivo.

This first volume provides a good perspective on current knowledge and research into growth factors and their action, and should be useful to investigators and teachers requiring such a perspective. However, if the series is to be of interest to those actively involved in this field of research it may be important to concentrate on articles which integrate studies on different factors and to shorten the publication time.

J. Taylor-Papadimitriou is a Staff Scientist at the Imperial Cancer Research Fund Laboratories, Lincoln's Inn Fields, London. 